\title{
The effect of retinal location on the magnitude of the Poggendorff illusion
}

\author{
S. M. GREIST and J. B. GRIER \\ Northern Illinois University, DeKalb, Illinois 60115
}

\begin{abstract}
The effect of retinal locus on the magnitude of the Poggendorff illusion was investigated. A significant illusion was found to occur in the fovea and in undiminished magnitude at the peripheral locations horizontally displaced from the fovea. No significant illusion was induced at the vertically displaced positions. It is suggested that the results obtained at the positions displaced from the fovea may be attributable to the refracting surfaces of the cornea, and that these findings lend support to an account of the Poggendorff illusion which emphasizes the significant involvement of peripheral mechanisms.
\end{abstract}

Previous investigations of the Poggendorff illusion (a tilted transversal interrupted by two parallel lines) have shown the illusory magnitude to be functionally related to certain variations in the stimulus configuration (Hill, 1971; Pressey, 1970; Pressey \& Sweeney, 1969, 1972; Restle, 1969; Weintraub \& Krantz, 1971), to be dependent upon particular subject characteristics (Pressey, 1967; Pressey \& Sweeney, 1970; Segall, Campbell, \& Herkovits, $1963)$, to be influenced by the nature of the subject's task (Cameron \& Steele, 1905; Coren \& Girgus, 1972; Novak, 1966), and to be largely resistant to destruction when viewed in stereoscope (Coren, 1970; Day, 1961; Schiller \& Weiner, 1962). This rather recent surge of interest in the Poggendorff illusion has produced a confusing and often contradictory conglomeration of experimental findings, and has shed little light on the mechanisms responsible for the perceived noncollinearity of the two transversal segments.

Several theories prevail as general explanations of visual illusions, and Over (1968) and Tayal (1972) have furnished extensive reviews. However, those theories which deal specifically with the Poggendorff illusion demand mention.

Chiang's (1968) diffraction theory contends that light diffraction, retinal blurring, and optical aberrations serve to displace the actual vertex toward the interior of the angle, thereby producing the misperceived intersection. This theory has been voluminously criticized (Coren, 1969; Cumming, 1968; Fame, 1970; Pressey \& den Heyer, 1968).

Theories which propose a central basis in accounting for the Poggendorff illusion are more numerous. Pressey's (1971) assimilation theory maintains that when an observer makes judgments on a series of magnitudes, the extreme magnitudes assume the properties of the average of entities. Gillam (1971) elaborated on the inappropriate constancy scaling theory held by Green and Hoyle (1963) and Gregory
(1968), and proposed a depth processing theory to account for the illusion. Both the inappropriate constancy scaling and depth processing theories contend that inappropriate depth cues are responsible for the Poggendorff illusion. Weintraub and Krantz (1971), in proposing a perceived orientation theory, have suggested a subjective geometry which predicts the intersection of lines. This subjective geometry, although analogous to the external geometry of the physical world, differs from it and thereby accounts for the perceived misalignment of the transversal segments.

The magnitude of some visual illusions may be dependent upon retinal location. With the horizontalvertical illusion, Pearce and Matin (1969) have shown an illusory decrement and eventual reversal with retinal eccentricity along the vertical axis. However, they observed no systematic changes in illusory magnitude along the horizontal axis. Holmes (1974), with the simultaneous Mueller-Lyer illusion, found no hemifield differences in illusory magnitude with horizontally displaced presentations of the figure.

The purpose of the present investigation was to examine the effect of retinal locus on the magnitude of the Poggendorff illusion.

\section{METHOD}

Four male and four female introductory psychology students served as subjects.

The Poggendorff figures were rules maintaining line width $(w=.5 \mathrm{~mm})$, parallel height $(\mathrm{h}=20 \mathrm{~mm})$, transversal segment length $(1=10 \mathrm{~mm})$, angle $\left(a=50^{\circ}\right)$, and parallel separation $(\mathrm{d}=10 \mathrm{~mm}$ ) constant.

The stimuli were presented using a tachistoscope. A small center fixation dot was maintained except when the Poggendorff figures were actually present. On each trial, the Poggendorff figure was presented at one of five possible locations: center, right, left, above, or below the fixation point. A 150 -msec exposure was employed to prohibit voluntary eye movements to bring the peripheral stimuli into the fovea. The stimuli presented foveally were centered around the fixation point and subtended 
about $1.25^{\circ}$. The peripheral stimuli were presented approximately $3.12^{\circ}$ from the fixation point.

For each retinal location, two series of stimuli were used: one in which the right transversal segment was varied in its point of parallel attachment and one in which the left transversal was varied. This was done in $1-\mathrm{mm}$ intervals from the point of objective collinearity to $5 \mathrm{~mm}$ above and below this point.

The experiment was divided into two sessions, each approximately $21 / 2 \mathrm{~h}$ in duration. Each subject was assigned a presentation order such that during one session the right transversal segment was varied for all retinal positions, and during the other session the left transversal was varied. The presentation order was counterbalanced with the sex of the subject.

A modified method of limits procedure was used for each of the five retinal positions. A unique initial attachment point was chosen for each position, and was either above or below the point of objective collinearity. The subject judged whether the variable transversal was above or below a projection of the stationary transversal. Judgments of equality were not permitted. Based on the subject's judgment, the next adjacent stimulus was presented. This process continued until a criterion of two successive reversals of judgment was reached. All viewing was done with the sighting dominant eye, the other was covered with a black optical patch.

Trials for the five different retinal positions were randomly interleaved so that on any given trial the subject could not predict the location of the stimulus to be presented. The subject was required to concentrate on the fixation point throughout both experimental sessions.

When al! five retinal locations had reached the criterion of two successive reversals, a presentation set was complete and a new starting point for each position was determined. Each session consisted of 15 such presentation sets, so that during the experiment that were 30 measurements of the magnitude of the Poggendorff illusion for each retinal position for each subject.

\section{RESULTS}

Prior to analysis, data collected for judgments of the right transversal segment were translated in direction to that of the left transversal judgments.

The mean illusory magnitude and corresponding

Table 1

Mean Illusory Magnitude and SD for each Retinal Position

\begin{tabular}{lll}
\hline Position & Mean & SD \\
\hline Center & .1696 & .0566 \\
Right & .2167 & .1080 \\
Left & .1904 & .0821 \\
Up & .0213 & .1120 \\
Down & .0417 & .0950 \\
\hline
\end{tabular}

Table 2

Summary of Hypotheses

\begin{tabular}{lrrr}
\multicolumn{1}{c}{ Contrast } & DF & F & P \\
\hline 1. Center & $1 / 7$ & 71.82 & $<.0001$ \\
2. Left & $1 / 7$ & 32.21 & $<.0008$ \\
3. Right & $1 / 7$ & 43.04 & $<.0004$ \\
4. Up & $1 / 7$ & .29 & n.s. \\
5. Down & $1 / 7$ & 1.54 & n.s. \\
6. Left-Right & $1 / 7$ & 1.65 & n.s. \\
7. Up-Down & $1 / 7$ & .35 & n.s. \\
8. Horizontal-Vertical & $1 / 7$ & 200.99 & $<.0001$ \\
9. Center-Horizontal & $1 / 7$ & 1.11 & n.s. \\
10. Centcr-Vertical & $1 / 7$ & 27.17 & $<.0013$ \\
\hline
\end{tabular}

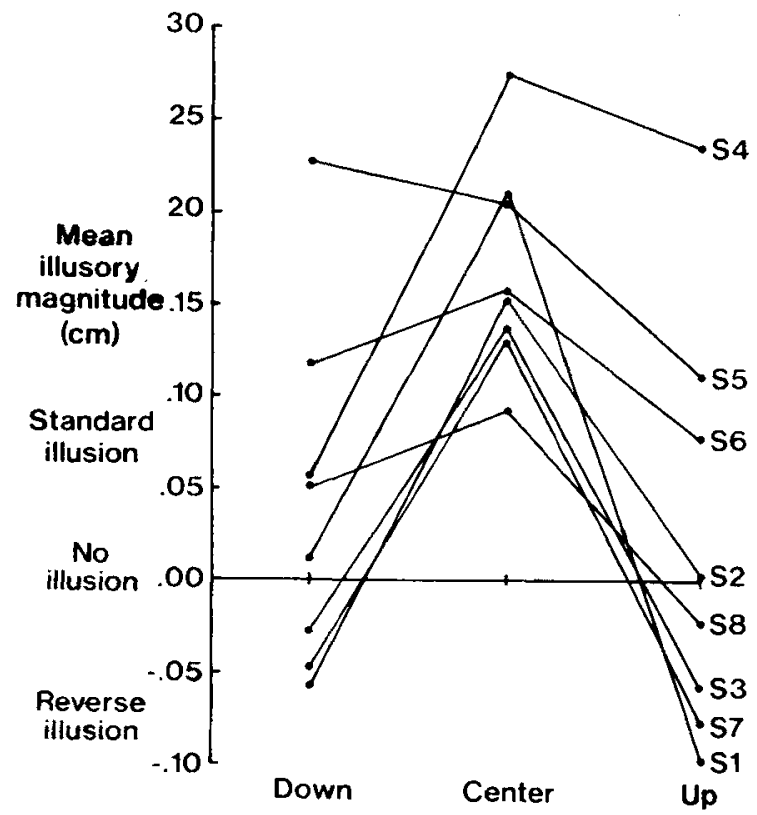

Retinal position

Figure 1. Mean illusory magnitude at the center and vertical positions for each subject.

standard deviation observed for each of the five retinal positions are presented in Table 1 .

A multivariate analysis of variance technique was used to test several hypotheses, and the results are summarized in Table 2.

The first five contrasts show that an illusion of significant magnitude occurred at the center, right, and left positions, whereas no significant illusion was induced at the up and down positions. Contrasts 6 and 7 demonstrate that no significant differences in illusory magnitude existed between the right and left positions, or between the up and down positions, respectively. The two horizontal positions (right, left) were found to differ significantly from the two vertical positions, as Contrast 8 shows. Contrasts 9 and 10 are not orthogonal to the preceding ones and were obtained in a later computer run. These contrasts illustrate that the center location, while significantly larger in magnitude than the vertical positions, did not differ from the horizontal ones in illusory magnitude.

The mean illusory magnitude observed for the center and vertical positions are plotted for each subject in Figure 1. More than half of the subjects exhibited a reverse illusion at either one or both of the vertical positions. The individual subject means are similarly presented for the center and horizontal locations in Figure 2.

Table 3 presents the mean illusory magnitude in blocks of five presentation sets for each retinal position. Each block represents five successive presentation sets of left transversal segment judgments and five translated presentation sets of right transversal 


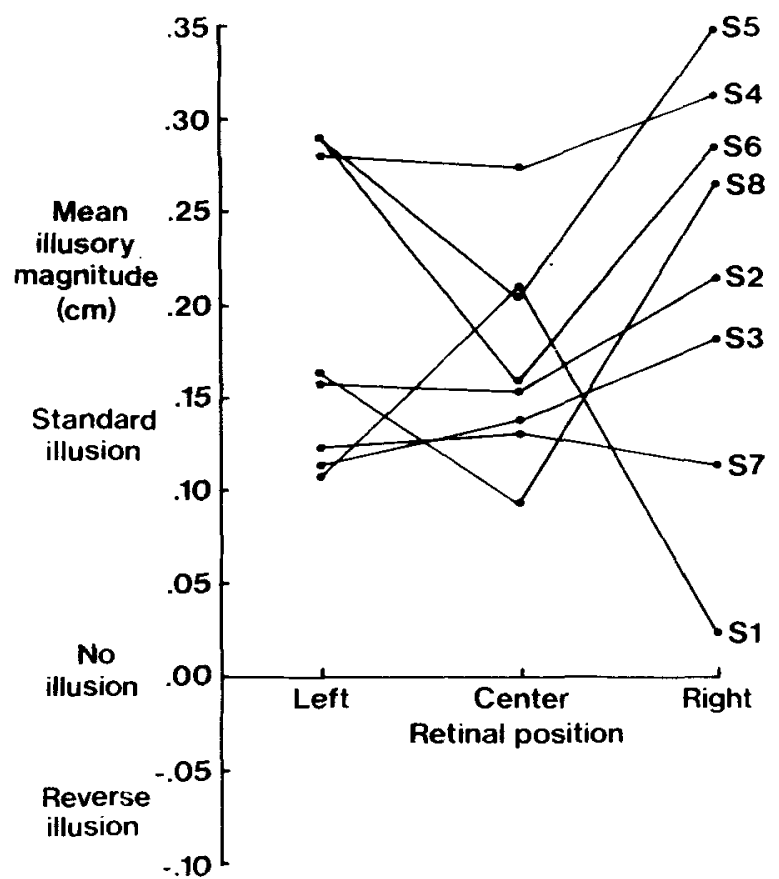

Figure 2. Mean illusory magnitude at the center and horizontal positions for each subject.

segment judgments which temporally corresponded during the two experimental sessions. Although a slight practice effect was observed, it appears that the pattern of results remained constant over time.

\section{DISCUSSION}

The results show a significant difference in the magnitude of the Poggendorff illusion between the horizontal and vertical periphery of the eye. The illusion induced in the horizontal periphery and the fovea did not significantly differ in magnitude, while no significant illusion occurred in the vertical periphery.

These results cannot be attributed to variations in the visual acuity of the peripheral regions of the retina, for Aulhorn and Harms (1972) have shown visual acuity to decrease in a radially symmetric fashion with retinal eccentricity.

In accounting for the illusory decrement and eventual reversal observed for the horizontalvertical illusion with vertical eccentricity, Pearce

Table 3

Mean Illusory Magnitude in Blocks of Five Presentation Sets for Each Retinal Position

\begin{tabular}{lccc} 
& & Block & \\
\cline { 2 - 4 } Position & $1-5$ & $6-10$ & $11-15$ \\
\hline Center & .1875 & .1650 & .1563 \\
Right & .2425 & .2113 & .1963 \\
Left & .2100 & .1663 & .1950 \\
Up & .0525 & .0025 & .0088 \\
Down & .0500 & .0225 & .0525 \\
\hline
\end{tabular}

and Matin (1969) contend that, due to the peripheral refracting surfaces of the eye, as vertical eccentricity increases, the vertical magnification of the target is reduced relative to the horizontal magnification. These circumstances predict an illusory decrement for both the horizontal-vertical and Poggendorff illusions. The obtuse angles of the Poggendorff figure have been found to be a critical feature of the illusion, not only with the degradation of the figure (Restle, 1969; Weintraub \& Krantz, 1971), but also as a determinant of illusory magnitude. Weintraub and Krantz (1971) have reported a systematic decrement in illusory magnitude as the obtuse angles of transversal attachment decrease. Although illusory magnitude has been found to increase linearly with the increasing separation of the parallels (Weintraub \& Krantz, 1971), it has been shown that an optimal distance between parallels exists beyond which illusory magnitude may decrease (Quina \& Pollack, 1973). No mean illusory effect was observed at the vertical positions tested in the present study, and several subjects exhibited an illusion of reversed magnitude at these positions.

Previous investigations of the Poggendorff illusion (Hill, 1971; Weintraub \& Krantz, 1971) have shown the magnitude of the illusion to decrease as the distance between the parallel segments is reduced, and to increase as the obtuse angles of the figure are increased. Pressey (1971), in observing that the illusory magnitude remains unaltered when the obtuse angles are increased simultaneous with a decrease in the distance between parallels, contends that the two contradictory trends cancel. With horizontal eccentricity, the dioptrics of the eye would cause a reduced horizontal magnification relative to the vertical, yet would produce no change in the magnitude of the Poggendorff illusion. This was indeed observed in the present study. However, the reduction in horizontal magnification would predict an increase in the magnitude of the horizontal-vertical illusion, yet this was observed in only one out of three subjects by Pearce and Matin (1969).

With the simultaneous Mueller-Lyer illusion, Holmes (1974) observed no difference in illusory magnitude when figures were presented in the right and left hemifields, and found her results not inconsistent with Pollack's $(1964,1969)$ two-process theory of visual illusions, which, in part, maintains that primary illusions are a function of stimulus variables and receptor properties. Indeed, the results of the present investigation are not inconsistent with a peripheral account of the Poggendorff illusion, for illusory magnitude was found to be equivalent in both right and left hemifields, as would be expected if the processing of the illusion occurs virtually at the retinal level. Furthermore, when coupled with Pearce and Matin's (1969) proposed involvement of the refracting surfaces of the cornea with retinal eccentricity, the results of the present study stress 
the importance of the stimulus-receptor interaction and its subsequent effect on the magnitude of the Poggendorff illusion. It is at least apparent in light of these findings that theories maintaining a central explanation of the Poggendorff illusion which exclude the involvement of peripheral receptor factors are at present inadequate.

While Sperry and his co-workers (Levy, Trevarthen, \& Sperry, 1972) have demonstrated the utility of the right-left hemifield dichotomy in investigating the hemispheric functions of the brain, the upper-lower hemifield dichotomy may have more significant perceptual implications. Breitmeyer, Julesz, \& Kropfl (1975) reported a significant difference in the time to detect depth between the upper and lower hemifields using random-dot stereograms, yet they observed no difference between the right and left hemifields. The necessity of further exploration of the upper-lower dichotomy is especially obvious in light of the present investigation, and will perhaps offer a useful tool in investigating the complexities of the peripheral mechanisms which may better account for the Poggendorff and similar illusions.

\section{REFERENCES}

Aulhorn, E., \& Harms, H. Visual perimetry. In D. Jameson \& L. M. HuRvich (Eds.), Handbook of sensory physiology, (VII/4). New York: Springer-Verlag, 1972.

Breitmeyer, B., Julesz, B., \& KRopfl, W. Dynamic random-dot stereograms reveal up-down anisotrophy and left-right isotropy between cortical hemifields. Science, 1975, 187, 269-270.

Cameron. E. H., \& Steele, W. M. The Poggendorff illusion. Psychological Monographs, 1905, 7, 83-111.

Chiang, C. A new theory to explain geometric illusions produced by crossing lines. Perception \& Psychophysics, 1968, 3, 174-176.

Coren. S. The influence of optical abberations on the magnitude of the Poggendorff illusion. Perception \& Psychophysics, 1969, 6, $185-186$.

Coren, S. Lateral inhibition and geometric illusions. Quarterly Journal of Experimental Psychology, 1970, 22, 274-278.

Coren, S., \& Girgus, J. S. Illusion decrement in intersecting line figures. Psychonomic Science, 1972, 26, 108-110.

Cumming, G. D. A criticism to the diffraction theory of some geometric illusions. Perception \& Psychophysics, 1968, 4, 375-376.

DAY, R. H. On the stereoscopic observation of geometric illusions. Perceptual and Motor Skills, 1961, 13, 247-258.

FamE, M. On the Poggendorff illusion: A note to Cumming's criticism of Chung Chiang's theory. Perception \& Psychophysics. $1970,8,112$.

Gillam, B. A. A depth processing theory of the Poggendorff illusion. Perception \& Psychophysics, 1971, 10, 211-216.

Green. R. T., \& Hoyle, E. M. The Poggendorff illusion as a constancy phenomenon. Nature, 1963, 200, 611-612.

Gregory, R. L. Visual illusions. Scientific American, 1968, 219, 66-76.
HiLl, A. L. Poggendorff illusion: Effects of intelligence, viewing distance, and space between the vertical lines. Psychonomic Science, 1971, 25, 71-72.

Holmes, S. The effects of ocular dominance and cerebral specialization on the magnitude of the Mueller-Lyer. Doctoral dissertation, University of Georgia, 1974.

Levy, J., Trevarthen, C., \& Sperry, R. W. Perception of bilateral chimeric figures following hemispheric deconnexion. Brain, 1972, 95, 61-78.

Novak, S. Effects of free inspection and fixation on the magnitude of the Poggendorff illusion. Perceptual and Motor Skills, 1966, 23, $663-670$.

Over, R. Explanations of geometrical illusions. Psychological Bulletin, 1968, 70, 545-562.

Pearce, D., \& Matin, L. Variation of the magnitude of the horizontal-vertical illusion with retinal eccentricity. Perception \& Psychophysics, 1969, 6, 241-243.

Pollack, R. H. Simultaneous and successive presentation of elements of the Mueller-Lyer figure and chronological age. Perceptual \& Motor Skills, 1964, 19. 303-310.

Pollack, R. H. Some implications of ontogenetic changes in perception. In D. Elkind \& J. Flavell (Eds.), Studies in cognitive development. Oxford: Oxford University Press, 1969.

Pressey, A. W. Field dependence and susceptibility to the Poggendorff illusion. Perceptual and Motor Skills, 1967, 24. 309-310.

Pressey, A. W. An explanation of the effect of tilt on the Poggendorff. Psychonomic Science, 1970, 18, 347.

Pressey, A. W. An extension of assimilation theory to illusions of size, area, and direction. Perception \& Psychophysics, 1971, 9, $172 \cdot 176$.

Pressey, A. W., \& den Heyer, K. Observations on Chiang's "new" theory of geometric illusions. Perception \& Psychophysics, $1968,4,313-314$.

Pressey. A. W., \& Sweeney, O. A variation of the Poggendorff illusion. Perceptual \& Motor Skills, 1969, 28, 883-886.

Pressey, A. W..\& Sweeney, O. Age changes in the Poggendorff illusion as measured by a method of production. Psychonomic Science, 1970, 19, 99-100.

Pressey, A. W.. \& Sweeney, O. Acute angles and the Poggendorff illusion. Quarterly Joumal of Experimental Psychology, 1972, 24, 169-174.

Quina, K., \& Pollack, R. H. Attraction of parallels as a function of intercontour distance. Perceptual \& Motor Skills, 1973, 36, 934.

Restle, F. Illusions of bent line. Perception \& Psychophysics, 1969, 5, 273-274.

SChiller, P.. \& Weiner, M. Binocular and stereoscopic viewing of geometric illusions. Perceptual \& Motor Skills, 1962, 15. $739-747$.

Segall, M. H., Campreli, D. T., \& Herkovits, M. J. Cultural differences in the perception of geometric illusions. Science, $1963,139,769-771$.

TAYAL, O. P. Visual processing: General principles for an interpretation of geometric illusions. Journal of General Psychology, 1972, 86, 93-115.

Weintraub, D. J., \& Krantz, D. H. The Poggendorff illusion: Amputations, rotations, and other perturbations. Perception \& Psychophysics, 1971, 10. 257-263.

(Received for publication November 14, 1975; revision accepted December $27,1976$. 